substrate processes," Proc. IEEE, vol. 57, no. 9, pp. 1532-1537, Sept. 1969.

[13] K. E. Bean and P. S. Gleim, "The influence of crystal orientation on silicon semiconductor processing," Proc. IEEE, vol. 57, no. 9, pp. 1469-1476, Sept. 1969.

[14] K. E. Bean and W. R. Runyan, "Dielectric isolation: Comprehensive current future," Electrochem. Soc. J., vol. 124, no. 1, pp. 5C-12C, Jan. 1977

[15] D. L. Kendall, Appl. Phys. Lett., vol. 26, no. 4, p. 195, Feb. 15, 1975.

[16] M. J. Declercq, "A new CMOS technology using anisotropic etching of silicon," IEEE J. Solid-State Circuits, vol. SC-10, no. 4, pp. 191-197, Aug. 1975.
[17] K. E. Bean, J. S. Crabbe, J. G. Hoffman, and F. D. Malone, "An improved radiation hardened JFET array," in Semiconductor Silicon 1973 (Electrochem. Soc.), H. R. Huff and R. R. Burgess, Eds.

[18] R. K. Smeltzer, D. L. Kendall, and G. L. Varnell, "Vertical multiple junction solar cell fabrication," in Conf. Rec. Tenth IEEE Photovoltaic Specialists Conf., p. 194, 1973.

[19] K. E. Bean, R. L. Yeakley, and T. K. Powell, "Orientation dependent etching and deposition of silicon," in ECS Extended $A b$ stracts Spring Meet. (May 1974), vol. 71-1.

[20] D. F. Weirauch, J. Appl. Phys, , vol. 46, p. 1478, 1975.

[21] E. Bassous and E. F. Baran, J. Electroch. Soc., vol. 125, no. 8, Aug. 1978.

\title{
Holographic Interference Lithography for Integrated Optics
}

\author{
WILLIE W. NG, CHI-SHAIN HONG, AND AMNON YARIV, FELLOW, IEEE
}

\begin{abstract}
In this paper, some of our recent work in the use of holographic interference lithography and various material removal techniques to corrugate thin-film optical waveguides are reviewed. The specific applications of these periodic thin-film devices in the distributed feedback and distributed Bragg reflector semiconductor laser, and as output grating couplers, are described. Recent results in the use and fabrication of chirped and curved-line gratings are also summarized.
\end{abstract}

\section{INTRODUCTION}

$I^{N}$ N INTEGRATED OPTICS we are interested in investigating the possibilities of incorporating optical components in thinfilm waveguides. The diffraction grating and the geodesic lens are examples in which thin-film technology and microfabrication techniques have made possible their incorporation into guided wave configurations. The gratings are fabricated by corrugating an interface of the waveguiding layer as shown in Fig. 1(a). Since, in most cases, the period of the corrugations required is of the order of a micrometer or less, conventional optical photolithographic techniques cannot be used for writing the grating lines. Instead, the grating masks have to be generated either by electron-beam lithography or holographic interference techniques. The latter is a maskless technique that records the interference fringes of two collimated interfering laser beams. In this paper we describe the state of the art of this technique and its application to some optoelectronic devices.

Since this Special Issue is devoted mostly to a review of the

Manuscript received February 29, 1978; revised June 15, 1978. This work was supported by the Air Force Office of Scientific Research and the National Science Foundation.

The authors are with California Institute of Technology, Pasadena, CA 91125 . various techniques of lithography, material removal, and deposition in the fabrication of devices, we will only sketch the basic principles of designing periodic thin-film waveguides. For details regarding the theoretical aspects, the interested reader is referred to [1]. This paper attempts to supplement [1], especially in the experimental aspects.

In Section III we describe in detail the fabrication of small period gratings by holographic interference. In Section IV we will describe the distributed feedback semiconductor (DFB) and distributed Bragg reflector (DBR) lasers. Such lasers utilize Bragg reflection as the feedback mechanism for laser oscillation and thus eliminate the necessity of terminating the semiconductor laser cavity at one of the cleavage planes of the $\mathrm{GaAs}$ substrate. The gratings also provide wavelength selectivity and stability [2] for the multiplexing of optical signals in optical communication. Section V describes the use of ionmilling for the fabrication of blazed gratings that can be used as output couplers for thin-film waveguides. And finally, in the last section, we describe some of our work in the use of curved wavefronts to generate grating lines with a variable period [3] ("chirped gratings") and novel curved line gratings that have focusing properties. The use of the former for wavelength demultiplexing has been demonstrated [4], [5].

\section{Wave Coupling in Corrugated Waveguides}

The case of Bragg reflection by a corrugated waveguide is shown in Fig. 1(b). The perturbation of the thin-film waveguide by the corrugations causes a contradirectional wave $A(z) e^{i \beta z}$, to be generated from the incident wave $B(z) e^{-i \beta z}$. The effectiveness of coupling is derived from a pair of coupled mode equations [6] and is characterized by a coupling parame- 
(a)

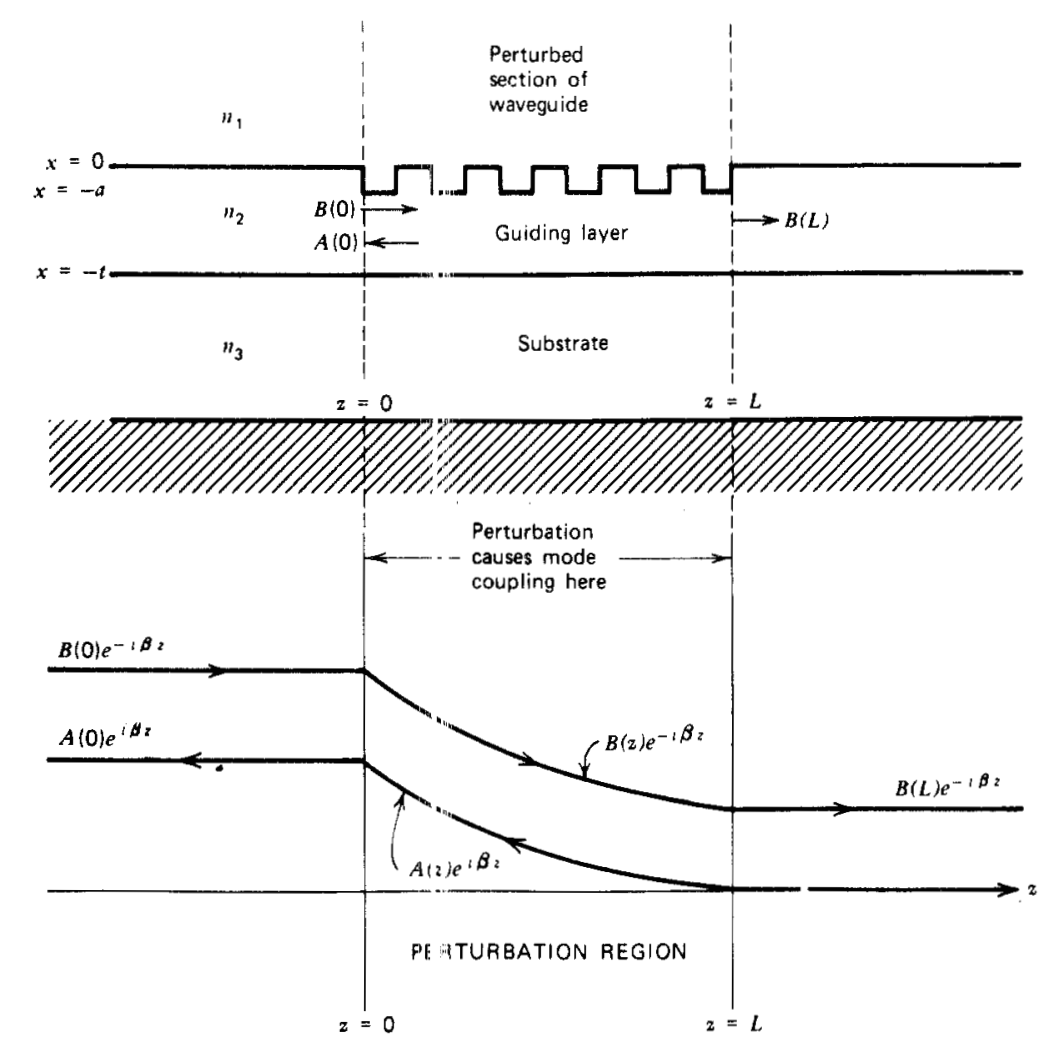

Fig. 1. (a) A corrugated waviguide section. The guiding layer with refractive index $n_{2}$ can be z layer of GaAs sandwiched by layers of GaAlAs $\left(n_{1}\right.$ and $n_{3}$ ). (b) Perturbation of the dielectric waveguide by the corrugations causes contradirectional mode coupling. The incident wave in $B(0) e^{-i \beta z}$, and the reflected wave is $A(0) e^{i \beta z}$.

ter $\kappa$. For square-wave corrugations

$$
\kappa=\frac{-\omega \epsilon_{0}}{4 \pi l} \int_{-\infty}^{\infty} \Delta n^{2}(x)\left[\varepsilon_{y}(x)\right]^{2} d x
$$

where the transverse mode profile is given by $\mathscr{E}_{y}(x)$, and $\Delta n^{2}(x)$, the grating function, is given for the case of squarewave gratings by

$$
\Delta n^{2}(x)= \begin{cases}n_{2}^{2}-n_{1}^{2}, & -a \leqslant x \leqslant 0 \\ 0, & \text { elsewhere. }\end{cases}
$$

Since the profile of the gratings is involved in the ovellap integral, we find that the profile and depth of the corrugation determine the magnitude of the contra-directionally coupled wave, or the reflectivity of the grating section. When the gratings are used as output couplers, the profile of the gratings comes into play in determining the relative intensities among the different orders of diffraction.

The design of a Bragg reflector or grating coupler for a dielectric waveguide starts with the determination of the period $(\Lambda)$ of the corrugations. This is found from the "phase-matching" or "momentum conservation" condition for the particular application. For example, the "phase-matching" condition fir a Bragg reflector (Fig. 2(a)) providing distributed feedback is

$$
2 \beta=l \frac{2 \pi}{\Lambda}
$$

where $l$ denotes the order of distributed feedback.

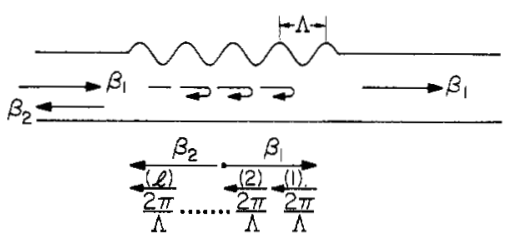

(a)

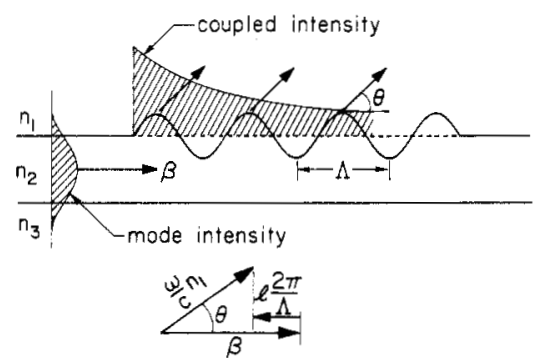

(b)
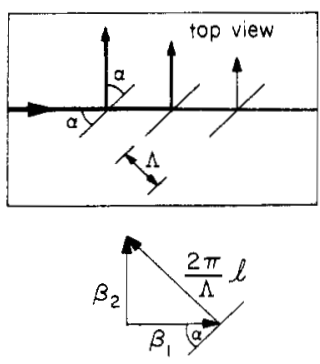

(c)

Fig. 2. (a) Bragg reflection. (b) Output coupling of guided wave. (c) Beam splitting of an incident wave. 


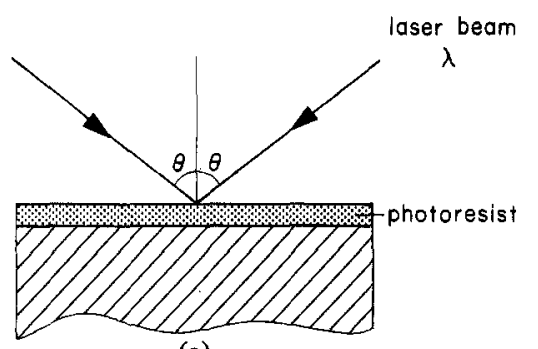

(a)

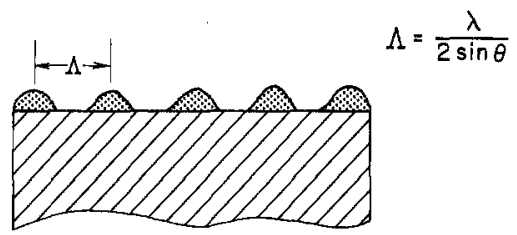

(b)

Fig. 3. A holographic setup for grating fabrication. (a) Exposure of photoresist by contradirectional laser beam derived from a common source. (b) Photoresist mask after development.

The phase matching condition for grating couplers and beam splitters is shown in Fig. 2(b) and (c), respectively. For the grating coupler

$$
\beta-\frac{\omega n_{1}}{c} \cos \theta=l\left(\frac{2 \pi}{\Lambda}\right) .
$$

For the beam splitter it can be easily shown that

$$
2 \beta \sin \alpha=l\left(\frac{2 \pi}{\Lambda}\right) .
$$

Since $\beta=(2 \pi / \lambda) n_{\mathrm{eff}}$, where $n_{\mathrm{eff}}$ is the effective index of refraction [7] of the waveguide, $\Lambda$ can be found from (1)-(3).

\section{Holographic INTERFERENCE TECHNIQUE}

In the holographic interference technique, the surface to be corrugated is spin-coated with photoresist (usually Az1350B). Two plane waves of the same frequency and of amplitudes $E_{1}$ and $E_{2}$ are incident upon a smooth surface at an angle $2 \theta$ as shown in Fig. 3(a). If the complex amplitudes of the waves across the surface are

$$
\begin{aligned}
& E_{1}=A \exp [-i k(z \sin \theta-x \cos \theta)] \\
& E_{2}=R A \exp [-i k(-z \sin \theta-x \cos \theta)-i \phi]
\end{aligned}
$$

respectively, the intensity on the surface is

$$
\left|E_{1}+E_{2}\right|_{x=0}^{2}=|A|^{2}\left\{1+R^{2}+2 R \cos 2[k z \sin \theta-\phi]\right\} .
$$

Hence, the intensity is modulated in the $z$ direction with a period $\Lambda=\lambda / 2 n(\sin \theta)$, where $n$ is the index of refraction of the medium in which the waves meet over the surface, and $\lambda$ is the vacuum wavelength of the waves. From the preceding expression, we see that we get maximum intensity modulation if $R=1$, or the intensity of the two intersecting beams is equal. Fig. 3(b) shows a photoresist mask after development.

The light source used most often in our work is a $\mathrm{He}-\mathrm{Cd}$ laser (4416- $\AA$ or $3250-\AA$ line) or an argon laser (4579- $\AA$ line). A spatial filtering arrangement (Fig. 4) consisting of a quartz lens and a $12.5-\mu \mathrm{m}$ pinhole is used as shown. The quality of a $\mathrm{He}-\mathrm{Cd}$ laser beam before and after spatial filtering is shown in Fig. 5. The "roughness" of the beam has high spatial Fourier

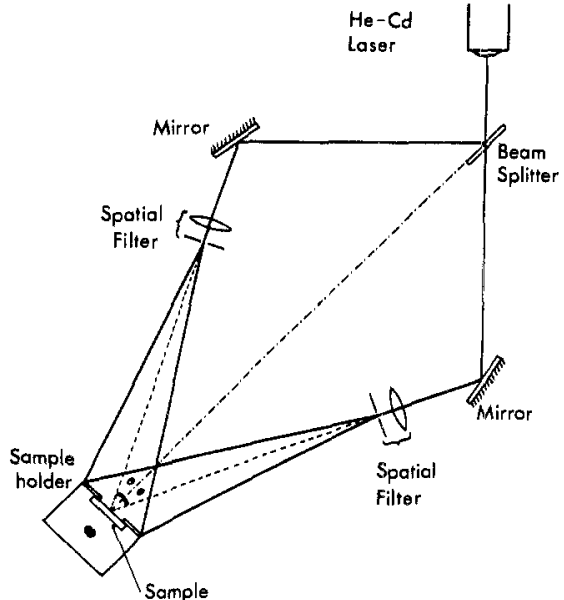

Fig. 4. Experimental setup for spatial filtering and exposure.

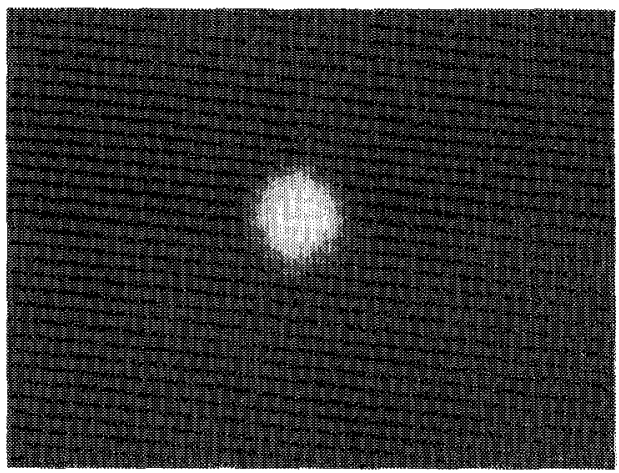

(a)

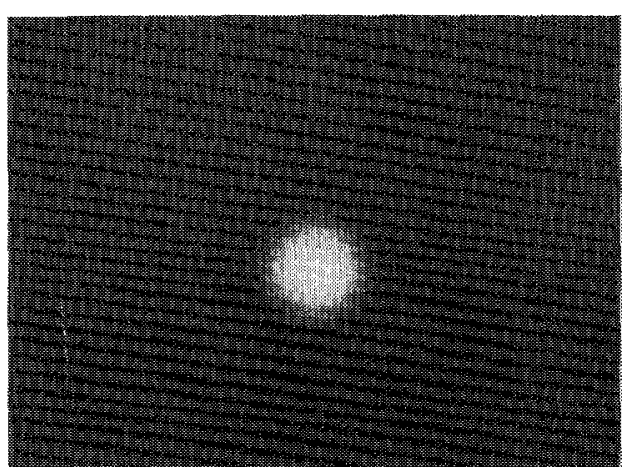

(b)

Fig. 5. (a) He-Cd beam before spatial filtering. (b) He-Cd beam after spatial filtering.

components at the focal plane of the lens, and is thus filtered out by the pinhole. The Fourier transform of the lowest order Gaussian mode is centered at the pinhole and is thus unaffected. Sometimes apertures are also placed between the beam splitter and the mirror to block unwanted reflections from the former.

From the expression for the period of the gratings, we see that the smallest period that can be obtained for the above experimental arrangement is $\Lambda=\lambda / 2$ when $\theta=90^{\circ}$. To obtain gratings with a period of $0.15 \mu \mathrm{m}$ or less, we immersed the sample to be corrugated in a high-index material [8], [9] as shown in Fig. 6(a) and (b). The smallest period that can be obtained is then $\lambda / 2 n$, where $n$ is the refractive index of the exposing medium. 
(a)

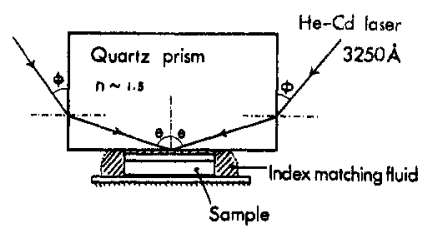

(b)

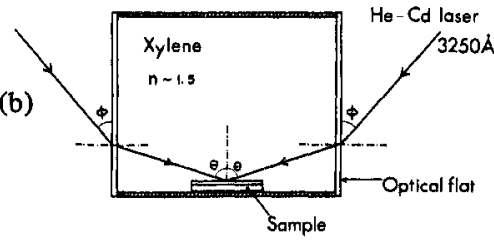

Fig. 6. (a), (b) Photographic exposure methods for short period.

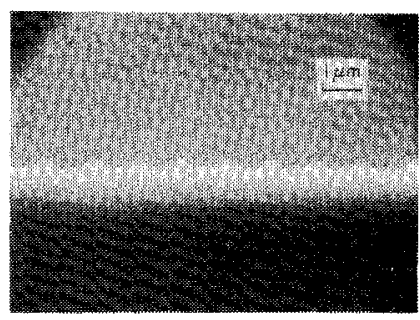

(a)

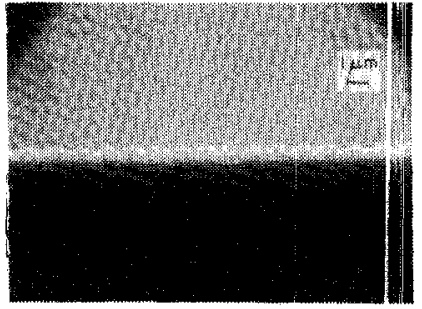

(b)
Fig. 7. (a) Grating profile obtained from wide photoresist mask openings using $\mathrm{C}_{2} \mathrm{H}_{4}(\mathrm{OH})_{2}, \mathrm{H}_{3} \mathrm{PO}_{4}$, and $\mathrm{H}_{2} \mathrm{O}_{2}(8: 1: 1)$. (b) Grating $p: 0-$ file obtained from narrow photoresist mask openings using $\mathrm{H}_{2} \mathrm{SC}_{4}$, $\mathrm{H}_{2} \mathrm{O}_{2}, \mathrm{H}_{2} \mathrm{O}$.

The substrate surface can be corrugated by either ion-milling [11] or by chemical etching [10] through the photoresist mask. Fig. $7($ a) shows the profile of gratings corrugated by chemical etching using $\mathrm{C}_{2} \mathrm{H}_{4}(\mathrm{OH})_{2}, \mathrm{H}_{3} \mathrm{PO}_{4}$, and $\mathrm{H}_{2} \mathrm{O}_{2}(8: 1: \cdots)$. The grating profile is determined by the anisotropic etching habit of the $\{100\} \mathrm{GaAs}$ substrate. The sides of the corruzations are the $\{111\}$ planes which have the slowest etching rate. Gratings etched by the $\mathrm{H}_{2} \mathrm{SO}_{4}-\mathrm{H}_{2} \mathrm{O}_{2}-\mathrm{H}_{2} \mathrm{O}$ system from photoresist masks, that have narrower groove openings than the crie shown in Fig. 7(a), are shown in Fig. 7(b). The chemical et :hing technique as opposed to ion-milling is found to introduce less nonradiative recombination centers in the operation of active devices, such as semiconductor lasers.

The photoresist that is usually used for the fabrication of gratings with periods of $0.5 \mu \mathrm{m}$ or less is diluted by photoresist thinner to a thickness of $1000 \AA$ (as checked by a Sloan Dektak). The use of thin photoresist was to make sure that the photoresist was developed down to the bottom after exposure. A technique which ensures this process was to expose and develop the photoresist at the same time [12]. Since the etching rate of photoresist by photoresist develoyer increases with the "light-dosage" that it receives (up to the bleaching level), the simultaneous exposure and developm nnt (SED) process reduces the exposure time for a given thickress of photoresist. This enhances the signal-to-noise ratio ind edge acuity of the resulting photoresist pattern. Theoret cal modeling of this technique was described in detail in [13] and [14].

In addition to chemical etching, ion-milling can also be used to corrugate the sample after photoresist development. T'his

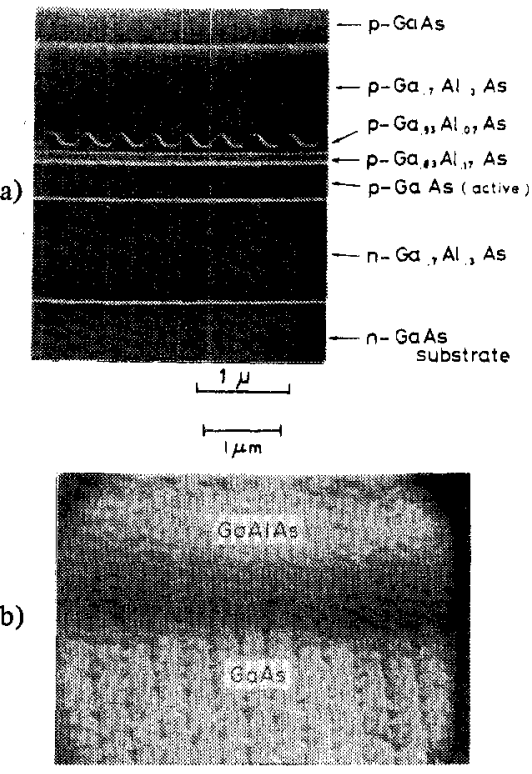

Fig. 8. (a) Device structure of a SC DFB laser. (b) Growth of $\mathrm{Ga}_{0.5}-\mathrm{Al}_{0.5}$ As on the corrugated surface of GaAs. Part of the $\mathrm{Ga}_{0.5} \mathrm{Al}_{0.5} \mathrm{As}$ has been etched away to show that the grating has not been etched away by meltback.

technique is especially useful in the fabrication of blazed gratings for use as output couplers, and will be discussed in Section V. Recently, it was found that to obtain high aspect ratio (groove depth/grating period) gratings with fine periods $(\sim 2500 \AA)$, ion beam etching can be used to advantage in combination with chemical etching [15]. Using ion-milling to remove the last few hundred angstroms of the undeveloped photoresist, gratings with a groove aspect ratio $>0.6$ were produced on GaAs.

\section{Distributed Feedback and Distributed BRAgG Reflector SEMiconductor LASERS}

The basic principle of the distributed feedback and distributed Bragg reflector laser was discussed in Section II. In this section we will discuss the various device structures that have been realized.

As mentioned previously, the effectiveness of the gratings in providing feedback is measured by the coupling constant, which is an overlap integral. Hence in the fabrication of distributed feedback or distributed Bragg reflector lasers, it is necessary to put the corrugations near the waveguiding layer of the laser [16]. However, the fabrication of a grating on the active layer caused interface recombination centers which made their room-temperature current threshold excessively high.

The problem has been overcome by the adoption of separate confinement (SC) heterostructures for the distributed feedback laser [17], [18], and the distributed Bragg reflector [19]-[22] laser, in which the gratings are put on the sides of the active region.

In the fabrication of the distributed feedback laser, it is necessary to regrow GaAlAs on a corrugated surface. This was reported in [23]. Fig. 8(b) shows such a regrowth which was performed at a temperature of $700^{\circ} \mathrm{C}$ and at a rapid cooling rate of $4-5^{\circ} \mathrm{C} / \mathrm{min}[24]$. Using this regrowth technique, CW 
(a)

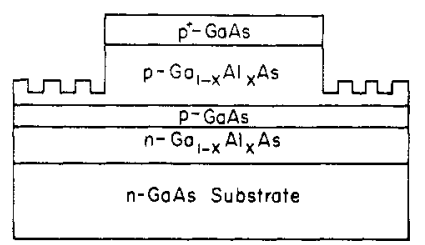

(b)

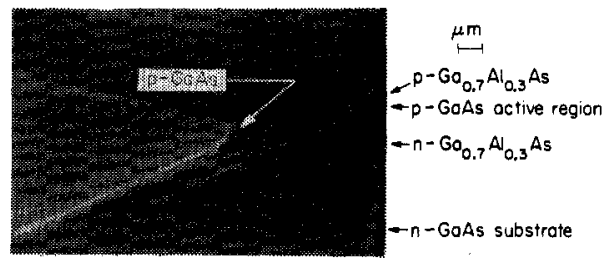

(c)

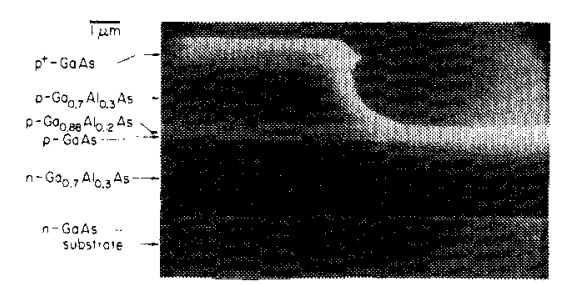

Fig. 9. (a) Schematic of an injection DHS DBR laser. (b) Shape of mesa when the mesa stripe is oriented in the (011) direction. (c) Mesa etched out by the use of "gold-etch" as a preferential etchant. The mesa stripe is oriented in the $(01 \overline{1})$.

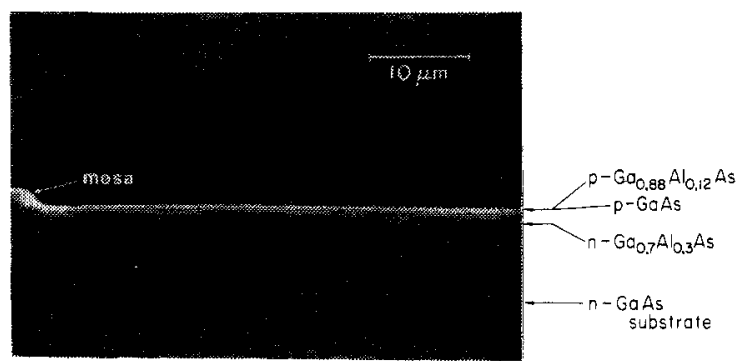

Fig. 10. Device structure after fabrication of gratings on opposite sides of the mesa.

separate confinement DFB laser has been fabricated with a structure as shown in Fig. 8(a) [18].

A schematic of the distributed Bragg reflector (DBR) laser [21] is shown in Fig. 9(a). It has a central pumped section with a pair of distributed Bragg reflectors on opposite sides of a mesa. Because the corruagtions are not fabricated on the part of the device where electron-hole recombination occurs, radiative recombination is not affected by the presence of the gratings.

Fabrication of this device started with the liquid-phase epitaxial growth of four [for a double-heterostructure (DH) DBR] layers or five (for a separate confinement DBR) layers of GaAs and GaAlAs on (100) oriented $n^{+}$GaAs substrates. Mesas are then etched out from the epi-layers. The mesas have sides in the $\{111\}$ plane and are shaped as shown in Fig. 9(b) and (c), depending on whether the mesa strip is oriented in the $(01 \overline{1})$ or $(0 \overline{1} \overline{1})$ direction. The mesa of the SC DBR (Fig. 9(c)) was etched out from the epi-layers by preferential etching and allows the gratings to be fabricated exactly on top of the low-loss $\mathrm{Ga}_{0.88} \mathrm{Al}_{0.12} \mathrm{As}$ layer (Fig. 10). The large optical cavity design of the separate confinement structure reduces the absorption loss of the unpumped section of this device.

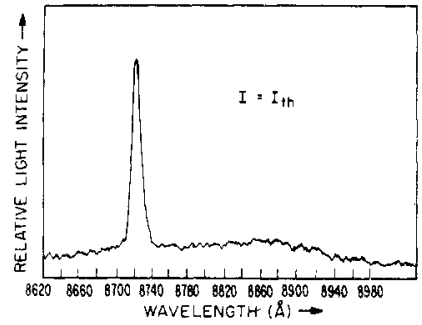

(a)

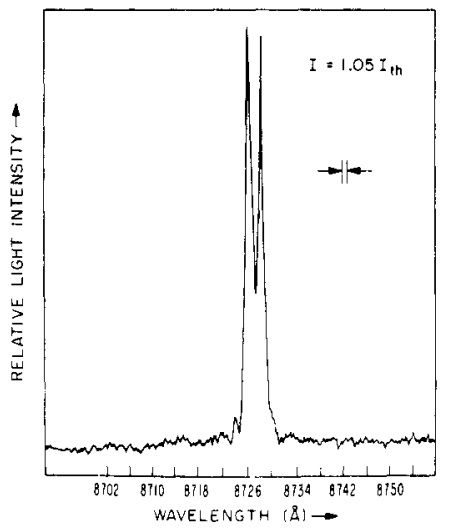

(b)

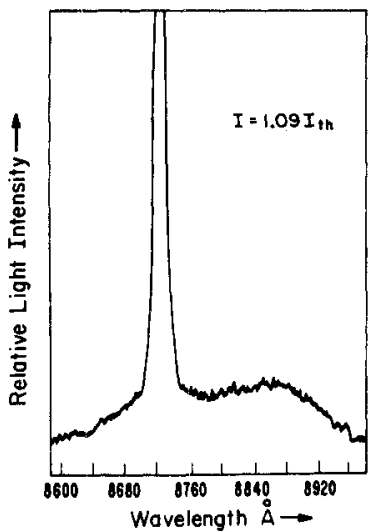

(c)
Fig. 11. (a) Low-resolution spectrum of the diode showing lasing at the short-wavelength side of the spontaneous emission peak. (b) Highresolution emission spectrum of the same diode at $I=1.05 I_{\mathrm{th}}$. (c) Emission spectrum of the diode at $I=1.09 I_{\mathrm{th}}$.

While the DBR laser was hindered by the presence of an unpumped section, device fabrication involves only one single step of liquid-phase epitaxy.

The effect of the corrugations on the emission spectrum is shown in Fig. 11(a), (c). Normally, diodes with an unpumped section lase at the long wavelength side of the spontaneous emission peak where the absorption is minimum. The wavelength selectivity of the gratings allows the diodes to lase even at wavelengths where the absorption is fairly high. A highresolution spectrum of the same diode (Fig. 11(b)) shows the presence of two modes at $I=1.05 I_{\mathrm{th}}$. The typical pulsed threshold current density of this kind of diode was $\sim 2-4$ $\mathrm{kA} / \mathrm{cm}^{2}$.

When the period of the gratings satisfy $\Lambda=4 \pi / \beta$, the grating provides Bragg reflection in the fourth order. In second order, it functions as an output coupler, providing low-divergence $\left(<1^{\circ}\right)$ broadside emission [25].

\section{Grating COUPLERS}

In the fabrication of grating couplers, the profile of the grating can be shaped so as to favor the coupling of the guided wave into air or the substrate. For a given waveguide and grating period $\Lambda$, it is possible to predict the blazing angle $\phi$ required to favor scattering into air. The direction of the beam coupled into air is determined by the phase-matching condition as discussed in Section II. The specific order that is coupled out will be enhanced if the face of the grating is inclined at an angle (Fig. 12(a)) such that the ray corresponding to the guided wave zig-zagging inside the guiding layer is reflected and refracted into the same direction as that predicted by the "phase-matching" condition. In Fig. 12(a) and (b) this im- 


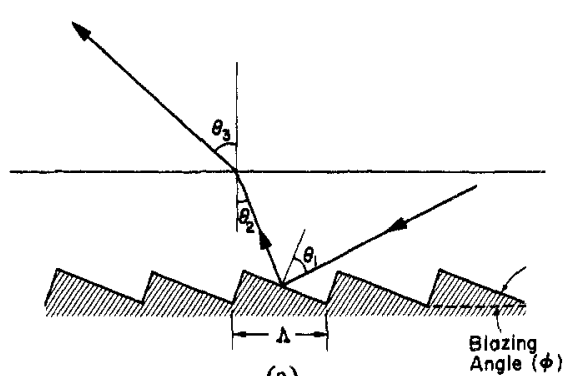

(a)

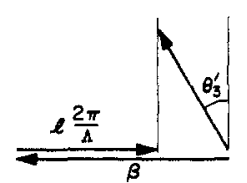

(b)

Fig. 12. (a) Ray optics picture of guided wave in a dielectric wavegude with blazed gratings at the substrate interface. (b) Phase-matching condition for output coupling.

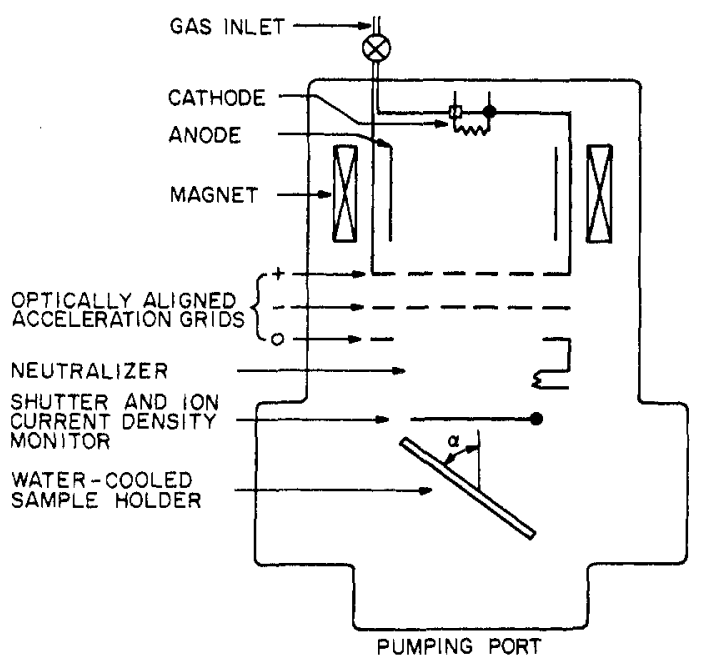

Fig. 13. Schematic diagram of the apparatus used for the fabrication of blazed gratings on $\mathrm{GaAs}$ by ion-milling.

plies $\theta_{3}=\theta_{3}^{\prime}$, where

$$
\sin \left(\theta_{3}^{\prime}\right)=\frac{\beta-l \frac{2 \pi}{\Lambda}}{\left(\frac{2 \pi}{\lambda}\right)}
$$

from phase matching. $\theta_{3}$ is related to the effective index $\left(n_{\text {eff }}\right)$ of refraction of the waveguide by the following equations from geometric optics considerations:

$$
\begin{aligned}
\theta_{1} & =\frac{\pi}{2}-\phi-\cos ^{-1}\left(n_{\text {eff }} / n_{\text {guide }}\right) \\
\theta_{2} & =\theta_{1}-\phi \\
\sin \theta_{3} & =\left(n_{\text {guide }}\right) \sin \theta_{2}
\end{aligned}
$$

where

$$
n_{\text {eff }}=\beta /\left(\frac{2 \pi}{\lambda}\right)
$$

and $n_{\text {guide }}$ is the index of refraction of the guiding layer.

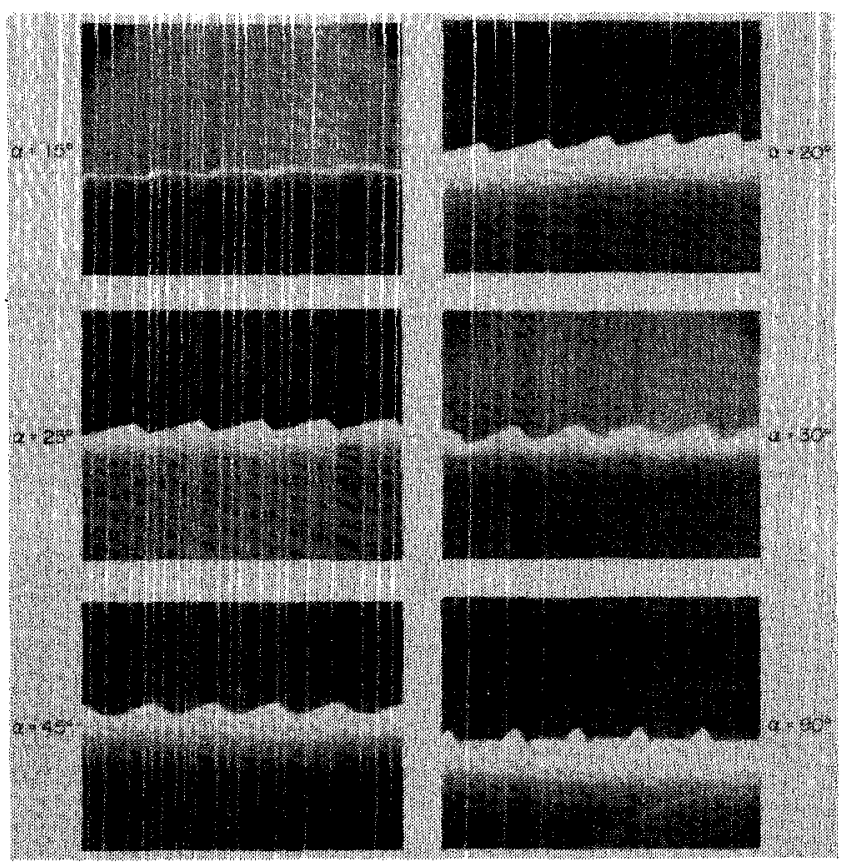

Fig. 14. Blazed gratings fabricated at various angles of ion-milling.

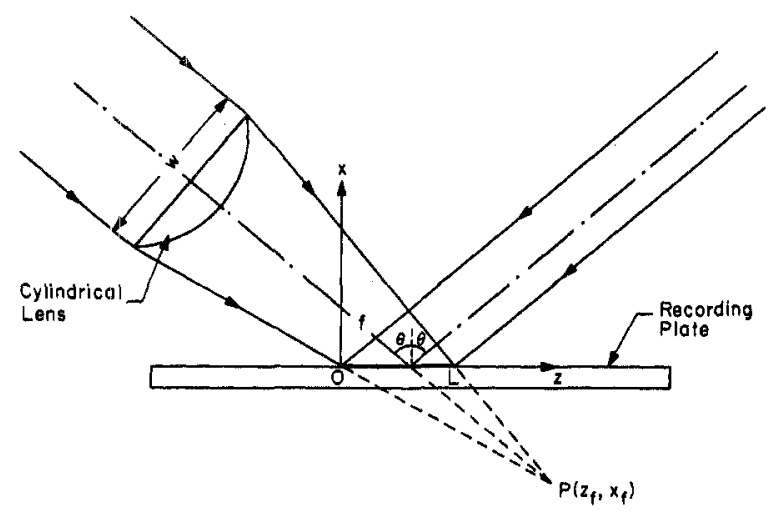

Fig. 15. Experimental setup for the fabrication of chirped gratings.

Experimentally, blazed gratings can be fabricated by ionbeam milling [11], [26] (Fig. 13). By inclining the substrate to be corrugated at an angle $\alpha$ to the ion beam, the photoresist shadows one side of the groove, thus forming grating with tooth shape that is asymmetric.

Fig. 14 shows the profiles of blazed grating for various values of $\alpha$. Accelerating voltage of $1 \mathrm{kV}$, ion-current density of 0.1 $\mathrm{mA} / \mathrm{cm}^{2}$, and milling time of 10 min were used during ionmilling. The period of the grating was $\sim 1 \mu \mathrm{m}$. The blazing effect was roughly evaluated by measuring the intensities of the +1 and -1 order diffracted beam from a normally incident beam (6328- $\AA$ He-Ne laser). With the laser polarization perpendicular to the grating grooves our best results correspond to an absolute diffraction efficiency $\eta$ of 54 percent, and an extinction ratio $\left(I_{1} / I_{-1}\right)$ of 227 .

\section{Chirped Gratings and Curved Gratings}

Gratings of variable [27] period, or chirped gratings, can be used as wavelength-selective beam splitters and hence can demultiplex an optical signal.

The setup used for the fabrication of chirped gratings is shown schematically in Fig. 15. Instead of interfering two 


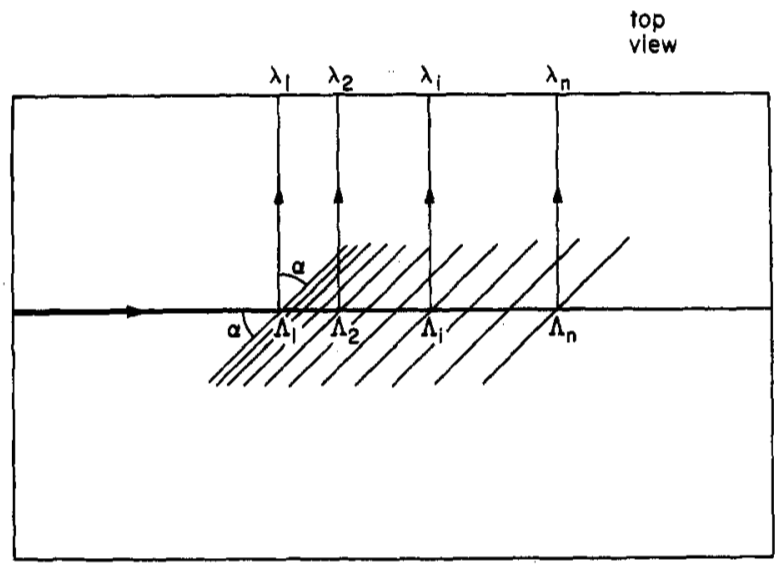

Fig. 16. Wavelength demultiplexing of an incident beam by a chirped grating beam splitter.

plane waves, the substrate to be corrugated is exposed to the interfering pattern of a collimated laser beam and a converging beam. The converging beam is generated by a cylindrical lens that is placed after the spatial filter. We can see physically (Fig. 15) that the period of the grating is larger at $L$ than at 0 because the angle of intersection between rays from the converging beam is smaller at $L$ than at 0 . The exact expression for $\Lambda(z)$ is $[27]$

$$
\Lambda(z)=\frac{\lambda}{\sin \theta+\frac{z_{f}-z}{\sqrt{\left(z_{f}-z\right)^{2}+x_{f}^{2}}} .}
$$

When a guided beam is incident on a corrugated region at an angle $\alpha$, the beam will be deflected according to Bragg's law $\left[\Lambda=\lambda / 2 n_{\text {eff }} \sin \alpha\right]$ by an angle $2 \alpha$ as discussed in Section II. If the grating has a variable period $\Lambda(z)$, then different wavelengths will be deflected from different locations of the corrugated waveguide, and will be separated spatially. (Fig. 16).

The interesting parameter in the design of the demultiplexer is the approximate number of resolvable wavelengths for a given length $\left(L_{T}=L / \sin \alpha\right)$ of grating with a chirping constant $\gamma$. Assuming that the period of the grating is chirped linearly in the direction $(z)$ of the incident beam $\left[\Lambda(z) \simeq \Lambda_{0}+\right.$ $(\gamma \sin \alpha) z$ ], the effective length $\left(L_{\text {eff }}\right)$ that will reflect light with a wavelength $(\lambda)$ can be derived from coupled mode theory [4]

$$
L_{\mathrm{eff}} \simeq \frac{\kappa_{l} \lambda^{2} l}{4 \pi \gamma n_{\mathrm{eff}}^{2} \sin ^{3} \alpha}
$$

where $l$ is the order of deflection and $\kappa_{l}$ is the coupling constant. The number of resolvable wavelengths in a length of $L_{T}$ is then

$$
N=\frac{L_{T}}{L_{\text {eff }}+w_{\text {beam }}}
$$

where $w_{b e a m}$ is the beamwidth of light in the waveguide.

Tien [28] recently proposed the fabrication of novel curved line gratings that possess focusing properties. Fig. 17 shows an optical arrangement used to generate the curved fringes. The curvature of the fringes is determined by $\theta$ and the distance of the recording plate from the focal plane. Specifically, the curvature is given by $1 /(2|\overline{O P}| \sin \theta)$. Hence to get fringes of

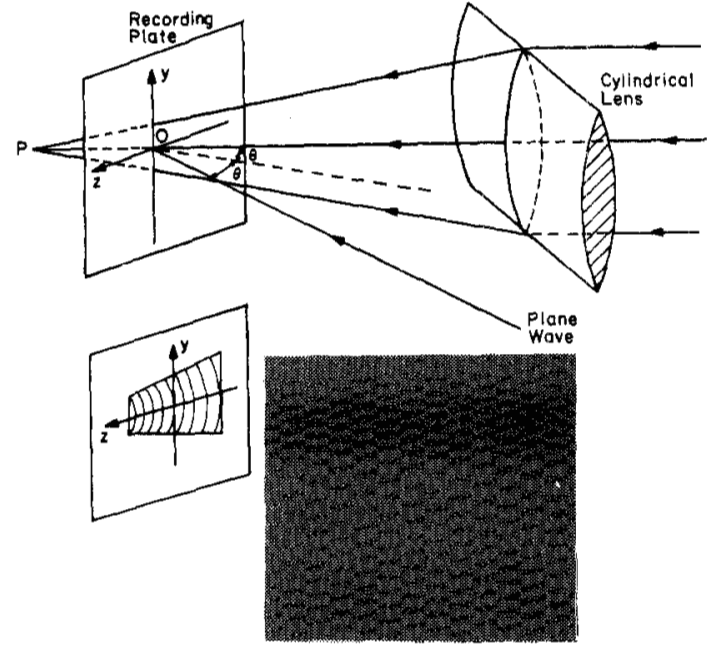

Fig. 17. Experimental setup for the fabrication of curved line gratings. The inset also shows some curved gratings fabricated on GaAs.

(a)

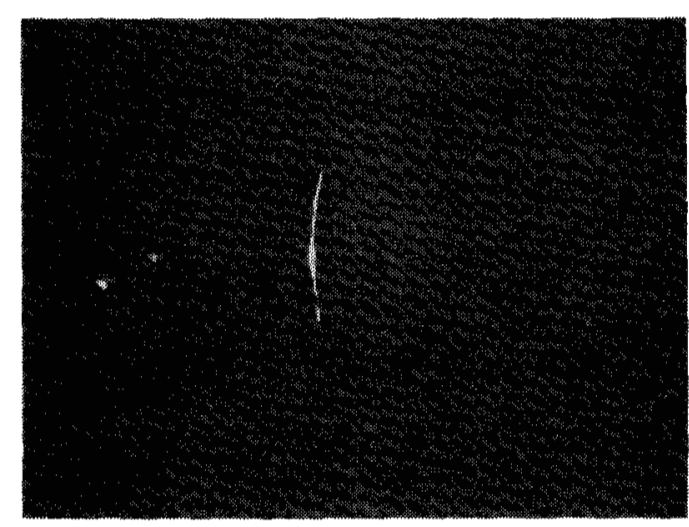

(b)

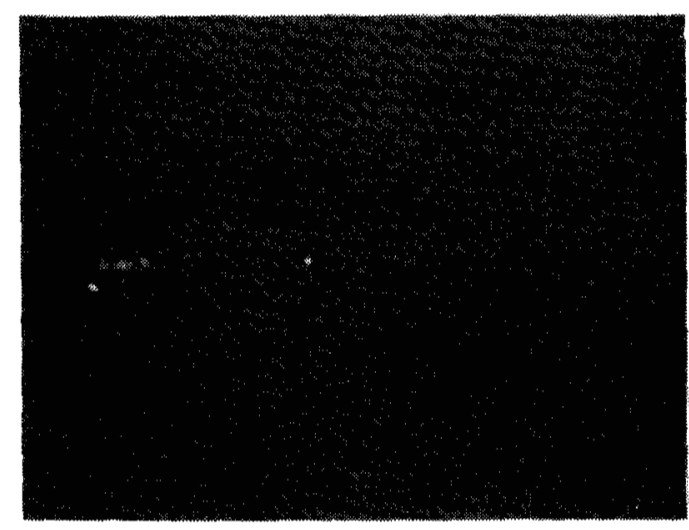

Fig. 18. (a) Far field of diffracted beam from curved gratings. (b) Far field of diffracted beam from gratings with no curvature.

small curvature, the recording plate must be placed very close to $P$. Unfortunately, in practice this introduces a variation of intensity in the $y$ direction because of the finite aperture of the cylindrical lens. The situation can be corrected to a certain extent by a spatially varying neutral density filter. The fact that the gratings thus fabricated are curved is demonstrated by the far field of the first-order diffracted beam from a $6328 . \AA$ $\mathrm{He}-\mathrm{Ne}$ beam that is incident normally on the grating (Fig. 18(a)). For comparison, Fig. 18(b) shows the far field of the diffraction from a grating with no curvature. 


\section{CONCLUSION}

In this paper we have attempted to summarize and revew some of our work in thin-film optoelectronic devices that rnake use of corrugated dielectric waveguides. We have thescribed the state of the art in the use of holographic intercirence techniques to generate gratings with curvature and variable period. The essential features of various material remc ial techniques, such as chemical etching and ion-beam milling, were described, and the advantage of each technique tias discussed in relation to semiconductor DFB lasers, DBR lasurs, and grating couplers. The fabrication of these gratings is compatible with thin-film deposition processes such as liquidphase epitaxy. The unique Bragg-reflection properties of thise corrugated thin-film structures at selected wavelengths mike them especially important in the multiplexing, demultiplexi : $g$, and filtering of optical signals in optical communication.

\section{ACKNOWLEDGMENT}

The authors wish to acknowledge H. W. Yen of the Hughes Research Laboratory for initiating some of the above wirk when he was with the California Institute of Technology.

\section{REFERENCES}

[1] A. Yariv and M. Nakamura, "Periodic structures for integraled optics," IEEE J. Quantum Electron., vol. QE-13, p. 233, $197 \%$.

[2] A. Aiki, M. Nakamura, and J. Umeda, "Frequency multiplesing light source with monolithically integrated distributed feedbick diode lasers," Appl. Phys. Lett., vol. 29, p. 506, 1976.

[3] A. C. Livanos, A. Katzir, and A. Yariv, "Fabrication of grating structures with variable period," Opt. Commun., vol. 20, p. 1.79, 1977.

[4] A. C. Livanos, A. Katzir, A. Yariv, and C. S. Hong, "Chirpedgrating demultiplexers in dielectric waveguides," Appl. Phys. Letr., vol. 30 , p. 519, 1977.

[5] M. Nakamura, "Integrated optics for wavelength division mtitiplexing," presented at Topical Meeting on Integrated and Guikled Wave Optics, Paper MB2, Salt Lake City, UT, 1978.

[6] A. Yariv, "Coupled-mode theory for guided-wave optics," IE:E J. Quantum Electron., vol. QE-9, p. 919, 1973.

[7] P. K. Tien, "Integrated optics and new wave phenomena in o] cal waveguides," Rev. Mod. Phys., vol. 49, p. 368, 1977.

[8] H. W. Yen, M. Nakamura, E. Garmire, S. Somekh, and A. Ya iv, "Optically pumped GaAs waveguide lasers with a fundamertal $0.11 \mu$ corrugation feedback," Opt. Commun., vol. 9, p. 35, 19 \% 3.

[9] C. V. Shank and R. V. Schmidt, "Optical technique for produc ag $0.1 \mu$ periodic surface structures," Appl. Phys. Lett., vol. : :3, p. $154,1973$.
[10] W. T. Tsang and S. Wang, "Profile and groove-depth control in GaAs diffraction gratings fabricated by preferential chemical etching in $\mathrm{H}_{2} \mathrm{SO}_{4} \cdot \mathrm{H}_{2} \mathrm{O}_{2}-\mathrm{H}_{2} \mathrm{O}$ systems," Appl. Phys. Lett., vol. 28, p. 44,1976

[11] S. Somekh and H. C. Casey, Jr., "Dry processing of high resolution and high aspect ratio structures in $\mathrm{GaAs}-\mathrm{Al}_{x} \mathrm{Ga}_{1-x}$ As for integrated optics," Appl. Opt., vol. 16, p. 126, 1977.

[12] W. T. Tsang and S. Wang, "Simultaneous exposure and development technique for making gratings on positive photoresist," Appl. Phys. Lett., vol. 24, p. 196, 1974.

[13] W. T. Tsang, "Theoretical modeling of the simultaneous exposure and development process of a positive photoresist," Appl. Opt. vol. 16, p. $1918,1977$.

[14] P. Agmon, A. C. Livanos, A. Katzir, and A. Yariv, "Simultaneous exposure and development of photoresist materials-An analytical model," Appl. Opt., vol. 16, p. 2612, 1977.

[15] L. F. Johnson, G. W. Kammlott, and K. A. Ingersoll, "Generation of periodic surface corrugations," Appl. Opt., vol. 17, p. 1165, 1978.

[16] M. Nakamura, K. Aiki, J. Umeda, A. Yariv, H. W. Yen, and T. Morikawa, "GaAs-Ga $-x \mathrm{Al}_{x}$ As double heterostructure distributed feedback diode lasers," Appl. Phys. Lett., vol. 25, p. 487, 1974.

[17] H. C. Casey, Jr., S. Somekh, and M. Ilegems, "Room temperature operation of low threshold separate confinement heterostructure injection laser with distributed feedback," Appl. Phys. Lett., vol. 27 , p. $142,1975$.

[18] K. Aiki, M. Nakamura, J. Umeda, A. Yariv, A. Katzir, and H. W. Yen, "GaAs-GaAlAs distributed-feedback diode lasers with separate optical and carrier confinement," Appl. Phys. Lett., vol. 27, p. 145,1975 .

[19] F. K. Reinhart, R. A. Logan, and C. V. Shank, "GaAs-Al $\mathrm{Ga}_{1-x} \mathrm{As}$ injection lasers with distributed Bragg reflectors," Appl. Phys. Lett., vol. 27, p. 45, 1975.

[20] W. T. Tsang and S. Wang, "GaAs-Ga $-\mathrm{Ga}_{1-x} \mathrm{Al}$ As double heterostructure injection lasers with distributed Bragg reflectors," $\mathrm{Appl}$. Phys. Lett., vol. 28, p. 596, 1976.

[21] W. Ng, H. W. Yen, A. Katzir, I. Samid, and A. Yariv, "Roomtemperature operation of GaAs Bragg-mirror lasers," Appl. Phys. Lett., vol. 29, p. 684, 1976.

[22] H. Namizaki, M. K. Shams, and S. Wang, "Large-optical-cavity $\mathrm{GaAs}-(\mathrm{GaAl}) \mathrm{As}$ injection laser with low-loss distributed Bragg reflectors," Appl. Phys. Lett., vol. 31, p. 122, 1977.

[23] M. Nakamura, K. Aiki, J. Umeda, A. Yariv, H. W. Yen, and T. Morikawa, "Liquid phase epitaxy of GaAlAs on GaAs substrates with fine surface corrugations," Appl. Phys. Lett., vol. 24, p. 466, 1974.

[24] W. Ng, I. Samid, and A. Yariv, unpublished.

[25] W. Ng and A. Yariv, "Highly collimated broadside emission from room-temperature GaAs distributed Bragg reflector lasers," Appl. Phys. Lett., vol. 31, p. 613,1977.

[26] T. Aoyagi, Y. Aoyagi, and S. Namba, "High efficiency blazed grating couplers," Appl. Phys. Lett., vol. 29, p. 303, 1976.

[27] A. Katzir, A. C. Livanos, J. B. Shellan, and A. Yariv, "Chirped gratings in integrated optics," IEEE J. Quantum Electron., vol. QE-13, p. 296, 1977.

[28] P. K. Tien, "Method of forming novel curved-line gratings and their use as reflectors and resonators in integrated optics," Opt. Lett., vol. 1, p. 64, 1977. 